\title{
Diversity of the Brain Dystrophin-Glycoprotein Complex
}

\author{
Kevin Culligan and Kay Ohlendieck* \\ Department of Pharmacology, Conway Institute for Biomolecular and Biomedical Research, \\ University College Dublin, Belfield, Dublin 4, Ireland
}

\begin{abstract}
Duchenne muscular dystrophy (DMD), the most common inherited neuromuscular disorder, is characterized by progressive muscle wasting and weakness. One third of Duchenne patients suffer a moderate to severe, nonprogressive form of mental retardation. Mutations in the DMD gene are thought to be responsible, with the shorter isoforms of dystrophin implicated in its molecular brain pathogenesis. It is becoming clear that region-specific variations in dystrophin isoforms delegate the composition of the dystrophinglycoprotein complex in brain, and hence, the function of the specific membrane assembly. Here we summarize the recent advances in the understanding of brain dystrophin, dystrophin-related proteins and dystrophin-associated proteins.
\end{abstract}

\section{INTRODUCTION}

The elucidation of the gene responsible for the primary defect in Duchenne muscular dystrophy (DMD) led to the discovery of its protein product, a membrane cytoskeletal protein termed dystrophin. The absence of this protein in patients afflicted with DMD leads to the characteristic dystrophic phenotype of progressive muscle weakness and wasting. Although primarily an inherited disorder of skeletal muscle, the disease also presents in cardiac muscle and diaphragm, as well as the peripheral and central nervous system $[1,2]$.

The $427 \mathrm{kd}$ dystrophin (Dp427) isoform, identified as a rod-shaped membrane cytoskeletal protein, can be divided into four distinct domains (Figure 1) $[1,2,3]$. The aminoterminal region interacts with cortical actin of the membrane cytoskeleton. Twenty-four spectrin-like repeats interrupted by four proline-rich hinge regions comprise the rod-domain, containing a second actin-binding site proximal to the amino-terminus. A cysteine-rich region containing a WWdomain protein-binding motif, together with an EF hand $\mathrm{Ca}^{2+}$-dependent region form the unique carboxy-terminal of Dp427. This domain is believed to bind dystrophin to a series of integral glycoproteins termed the dystrophinassociated proteins (DAPs) $[1,2,3]$. The carboxy-terminus also contains coiled-coil protein-binding motifs (Figure 1), shown to interact with the syntrophin protein family, and the dystrobrevin family of dystrophin-related/dystrophinassociated proteins. This group of proteins recruits signalling elements such as the neuronal nitric oxide synthase (nNOS) and Grb2, and is also implicated in the clustering of ion channels such as voltage-gated $\mathrm{Na}^{+}$-channels $[3,4]$.

A chromosome 6-encoded autosomal homologue of dystrophin named utrophin (Figure 1) has also been shown to interact with the DAPs [3]. Utrophin is localized to the neuromuscular junction [2], where interactions of utrophin with the DAPs, rapsyn and agrin lead to clustering of nicotinic acetylcholine receptors at the postsynaptic membrane in the junctional folds. The tertiary structure of utrophin is similar to that of dystrophin, however one less spectrin-like repeat results in a $395 \mathrm{kd}$-protein transcript. Utrophin contains a unique amino-terminus, with an absence of a similar actinbinding site of the dystrophin rod domain [2]. Binding to $\beta$-dystroglycan is also mediated by a WW domain, with the interaction stabilized by an adjacent EF-hand region [5]. An interaction of utrophin with the cytoplasmic components associated with dystrophin has also been demonstrated, mediated by coiled-coil motifs [3].

\section{BRAIN DYSTROPHIN-GLYCOPROTEIN COMPLEX}

Brain abnormalities, presented in the form of a moderate to severe, nonprogressive mental retardation, are manifest in one third of the patients affected by DMD [6]. The abnormality is evident as developmental cognitive and behavioural abnormalities, including deficits in overall and verbal IQ, as well as attention deficits and impaired short-term memory processing [6,7]. Analysis of the dystrophin gene revealed the presence of eight different promoters scattered throughout the gene, driving tissue-specific distribution of full-length dystrophins and several shorter isoforms. Full-length Dp427 isoforms were discovered in brain, as well as three shorter C-terminal transcripts. Downstream promoters drive the expression of a $140 \mathrm{kd}$ isoform termed Dp140, and the $71 \mathrm{kd}$ major brain isoform Dp71 (see Figure 1 and Table 1) [2]. Common to all the brain dystrophin isoforms is the inclusion of the WW-motif and EF-hand region, as well as the coiled-coil regions. Therefore, a link is maintained to the integral membrane protein $\beta$-dystroglycan, as well as to the members of the cytoplasmic component of the dystrophinglycoprotein complex (DGC). The shorter isoforms lack the actin-binding domains of the muscle dystrophin isoform, although the $\mathrm{N}$-terminus of Dp71 contains a unique 


\begin{tabular}{|c|c|c|}
\hline $\begin{array}{l}\text { Amino- } \\
\text { terminus }\end{array}$ & Rod domain & $\begin{array}{l}\text { Cysteine-rich } \\
\text { domain }\end{array}$ \\
\hline
\end{tabular}
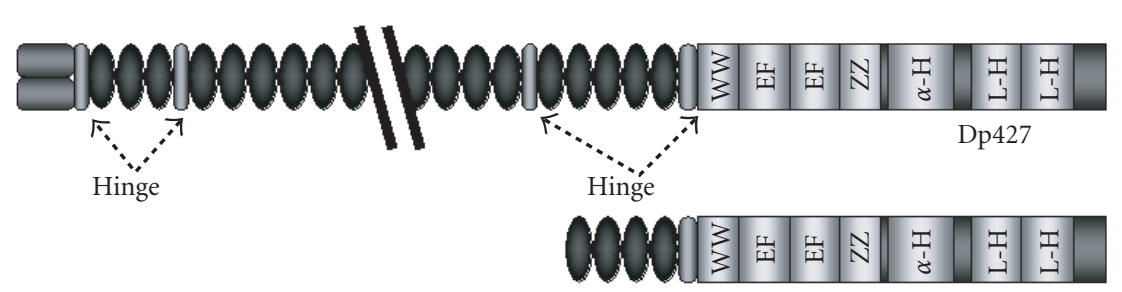

Dp140
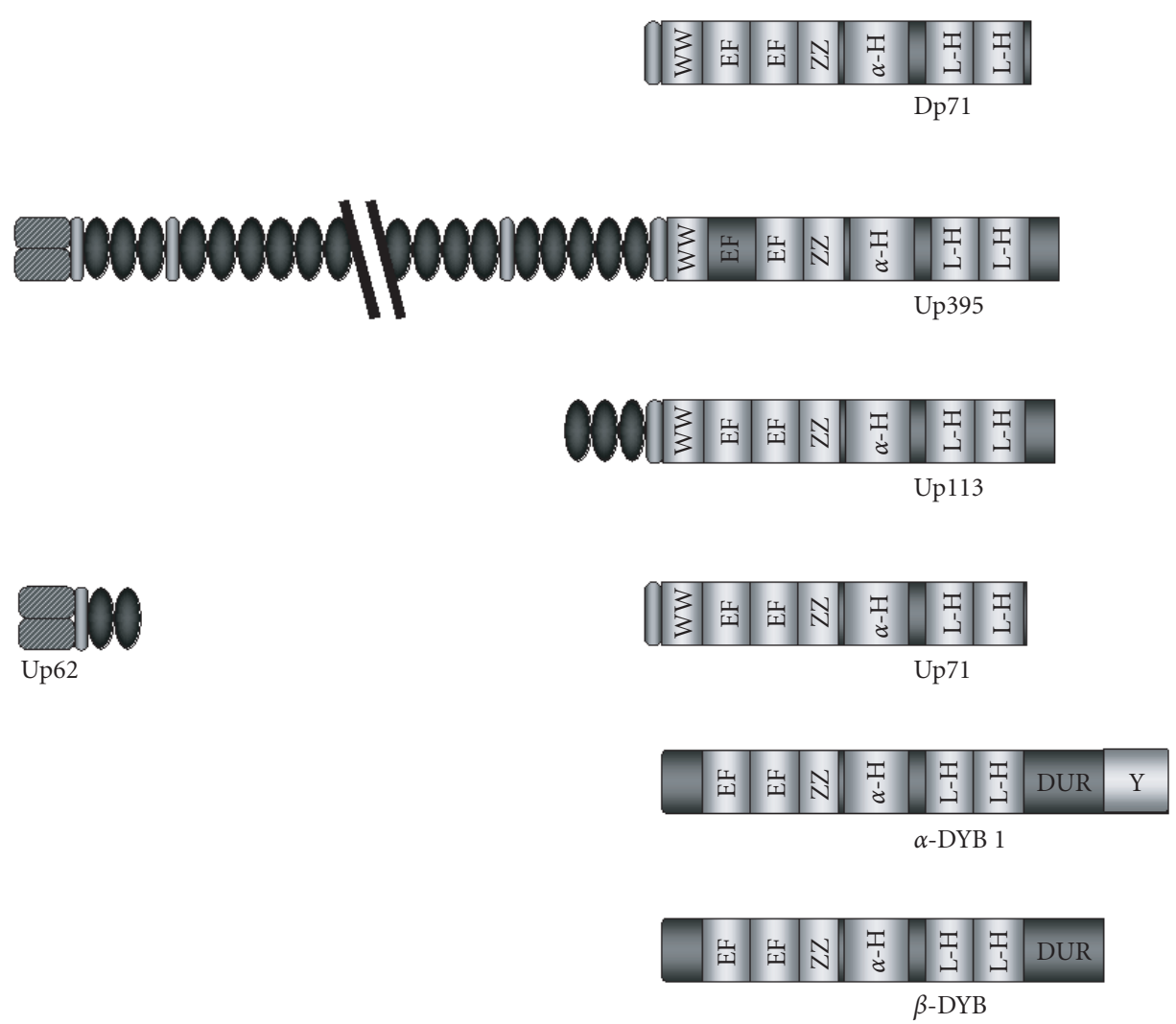

FIGURE 1. Dystrophin and the dystrophin-related proteins specific to brain. Schematic representation of dystrophin and the dystrophin-related proteins, and the similarities shared by each protein. Shown are the four distinct regions of dystrophin: the amino-terminus, the rod-domain interrupted by the four proline-rich hinge regions, the cysteine-rich domain, and the carboxy-terminus. Abbreviations used: Hinge: hinge regions, Cysteine: cysteine-rich domain, WW: PPxY-binding WW motif, EF: $\mathrm{Ca}^{2+}$-dependent twin EF-hand motifs, ZZ: $\mathrm{Zn}^{2+}$-dependent zinc fingers, $\alpha$-H: $\alpha$-helical domain, L-H helical leucine heptads which makeup the coiled-coil domain, DUR: dystrobrevin-unique region, Y: region of tyrosine phosphorylation.

N-terminal actin-binding domain. The brain DGC consists of four main components: the dystroglycan subcomplex, a dystrophin or utrophin isoform, a dystrobrevin isoform, and a syntrophin subcomplex.

\section{DYSTROGLYCAN SUBCOMPLEX}

The backbone of the brain DGC is the transmembrane link generated by the presence of $\alpha$ - and $\beta$-dystroglycan. These proteins act to form an integral sarcolemmal linkage, localizing dystrophin to the subsarcolemmal region. A proline-rich region at the extreme carboxy-terminus of $\beta$-dystroglycan mediates this interaction with dystrophin and its isoforms. As with the muscle complex, binding is mediated by the WW domain of dystrophin, stabilized by the adjacent EF-hand region [5]. However, the lack of sarcoglycans in the brain, in particular $\delta$-sarcoglycan, which is found in close proximity to $\alpha$ - and $\beta$-dystroglycan in muscle, has led to the speculation that a more tenuous binding exists between the dystroglycans and dystrophin [8]. Chemical crosslinking of brain $\beta$-dystroglycan results in the stabilization of a high-molecular mass complex (Figure 2a) [2] . On the other hand, perfluoro-octanoic acid polyacrylamide gel electrophoresis (PFO-PAGE) analysis, performed as described in reference [9], indicates that the monomeric form of $\alpha$-dystroglycan prevails in brain over the oligomerized 
TABLE 1. Location of the brain isoforms of dystrophin throughout the brain.

\begin{tabular}{|c|c|}
\hline Dystrophin isoform & Cellular location \\
\hline Dp427-M & Glia and vascular endothelial cells \\
\hline Dp427-C & Cortical and hippocampal (CA 1-3 region) pyramidal neurons \\
\hline Dp427-P & Fetal cerebral cortex and mature cerebellar purkinje cells \\
\hline \multirow[t]{4}{*}{ Dp140 } & Astroglial processes throughout the neuropil during development \\
\hline & Leptomeningeal surfaces and along penetrating blood vessels \\
\hline & Ensheathing the olfactory nerve \\
\hline & Neurohypophysis \\
\hline \multirow[t]{2}{*}{ Dp116 } & Late human fetal brain \\
\hline & Caudate/putamen region \\
\hline \multirow[t]{11}{*}{ Dp71 } & Early embryonic stem cells \\
\hline & Mesenchymal-epithelial interactions \\
\hline & Embryonic midbrain and hindbrain \\
\hline & Perinatal brain at stages of terminal neural differentiation \\
\hline & Early embryonic forebrain continuing until adult life \\
\hline & Cerebral cortex \\
\hline & Granule neurons of dentate gyrus of hippocampus \\
\hline & Neocortial regions (neurogenesis and synaptic plasticity) \\
\hline & Olfactory bulb \\
\hline & Pituitary gland \\
\hline & Retina \\
\hline
\end{tabular}

forms seen in skeletal and cardiac muscle (Figure 2b). The recent analysis of the dystrophic mdx mouse model demonstrated abnormal oligomerization of the neuronal Dp71 isoform suggesting its involvement in the pathophysiological mechanisms underlying abnormal brain functions [10].

Structural analysis of $\beta$-dystroglycan has indicated numerous possibilities for the interaction of $\beta$-dystroglycan with signalling and second-messenger proteins. Two WWbinding domains have been identified from the sequence of $\beta$-dystroglycan, although only one ligand for these sites is known. Several SH3-binding domains have also been identified, although only the intracellular adapter protein Grb2 has been shown to interact with $\beta$-dystroglycan. The identification of the Grb2-interacting protein focal adhesion kinase as a component of the DGC, a known phosphorylator of the DGC, still remains elusive $[5,8]$.

The extracellular matrix component $\alpha$-dystroglycan, a $156 \mathrm{kd}$ heavily glycosylated protein interacts with the aminoterminal region of $\beta$-dystroglycan. In muscle, $\alpha$-dystroglycan has been demonstrated to act as a receptor for several different ligands. Bindings to proteins such as the $\alpha 2$-chain of laminin-1, 2 and 8 have been demonstrated, as well as perlecan and agrin [8]. Implications have been made for the interaction of $\alpha$-dystroglycan with pathogenic viruses such as leprosy and lassa fever viruses [8]. Recently, three different glycosylation states of $\alpha$-dystroglycan have been identified in muscle, leading to the postulation that the extent of glycosylation of $\alpha$-dystroglycan plays a key role in the formation of complexes with extracellular proteins [11]. With the binding sites for laminin and perlecan in close proximity, differential glycosylation of $\alpha$-dystroglycan may lead to exposure of the relevant ligand-binding site leading to selective recruitment of extracellular binding proteins.

Quantitative studies on laminin, perlecan, and agrin in brain tissue indicate that the levels of these proteins expressed in brain are less significant than that of $\alpha$ dystroglycan. Agrin has been implicated to interact with Dp71 in astrocytes, while laminin has been localized to the glial-vascular interphase of the capillary basement membrane. Recently, a presynaptic neuronal protein involved in cell aggregation was identified as a neuron-specific $\alpha$ dystroglycan-binding protein. The polymorphic cell-surface proteins, $\alpha$ - and $\beta$-neurexins, have been demonstrated as binding partners for neuronally expressed dystrophin through an interaction with $\alpha$-dystroglycan. The interaction links the neuronal postsynaptic membrane through $\alpha$ dystroglycan with the presynaptic membrane through the neurexins, mediating cell aggregation [12]. As diagrammatically shown in Figure 3, it is becoming increasingly clear that different DGCs exist in the central nervous system [13].

\section{BRAIN DYSTROPHINS}

Localization studies have determined a neuronal distribution of Dp427, being associated with the postsynaptic density. Three full-length dystrophin isoforms have been established, resulting from different promoters, differing only in 


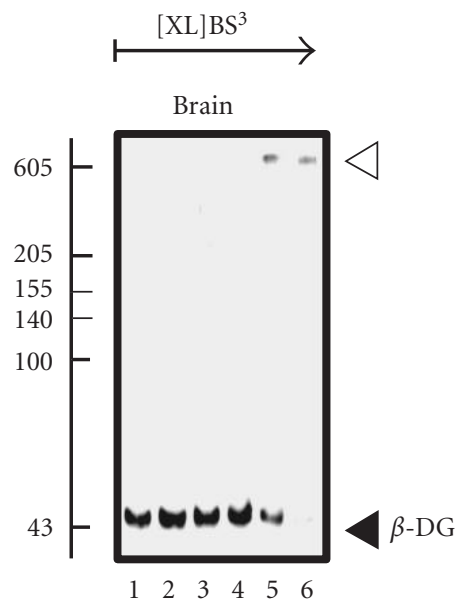

(a) SDS-PAGE

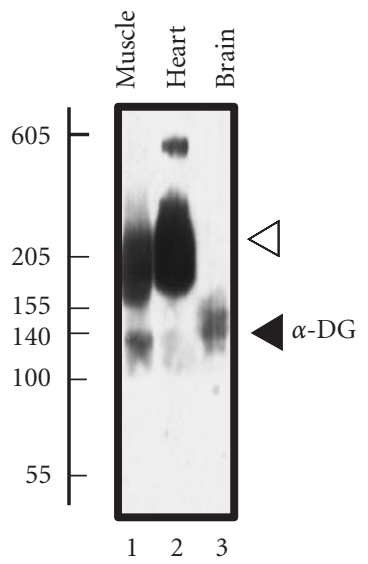

(b) PFO-PAGE
FIGURE 2. Analysis of the brain dystroglycan complex. Panel (a) shows the chemical cross-linking analysis of $\beta$-dystroglycan ( $\beta$-DG) of apparent $43 \mathrm{kd}$ in normal mouse brain using the hydrophilic probe bis-sulfosuccinimidylsuberate $\left(\mathrm{BS}^{3}\right)$ and sodium dodecyl sulfate polyacrylamide gel electrophoresis (SDS-PAGE). Detection of this sarcolemmal protein was achieved by immuno-decoration with monoclonal antibody NCL-b-DG [10]. Lanes 1-6 represent $0,25,50,100,200$, and $300 \mu \mathrm{g} \mathrm{BS} / \mathrm{mg}$ protein, respectively. Increasing concentrations of crosslinker (XL) clearly stabilized a highmolecular-mass complex demonstrating the oligomeric status of brain $\beta$ dystroglycan. In panel (b), is shown the perfluoro-octanoic acid polyacrylamide gel electrophoresis (PFO-PAGE) [9] analysis of $\alpha$-dystroglycan ( $\alpha$ DG). Lanes 1 to 3 represent mouse skeletal muscle, cardiac muscle, and brain microsomal membranes, respectively. The highly glycosylated, extracellular protein $\alpha$-dystroglycan was immuno-decorated employing monoclonal antibody $\mathrm{VIAl}_{2}$ [10]. Compared to skeletal and cardiac muscle, the dystroglycan sub-complex was not preserved in brain by this method, indicating a more tentative dystroglycan binding in the central nervous system. The position of monomers and oligomers is marked by closed and open arrow heads, respectively. The relative position of molecular mass markers (in $\mathrm{kd}$ ) is indicated on the left of blots.

their amino-terminal makeup and their cellular location. The shorter Dp140 isoform of dystrophin is comprised of the distal part of the rod domain, and an identical carboxyterminus to that of full-length dystrophin. Expression profiles of Dp140 show an ubiquitous distribution throughout the brain, being localized to penetrating cerebral microvasculature and expressed predominantly during fetal development [6].

The major dystrophin protein product in brain, Dp71, is expressed in astrocytes and glial cells $[14,15]$. Lacking the actin-binding domain of full-length dystrophin and the spectrin-like repeats, Dp71 retains the carboxy-terminal region, the cysteine-rich domain, and part of the hinge region proximal to the cysteine-rich domain. The transmembrane link is maintained by an interaction with $\beta$ dystroglycan, which in turn interacts with $\alpha$-dystroglycan. Recently, two alternative splicing sites were determined. Although one of the sites has no effect on the protein, the other site, which omits exon 78, results in the addition of a hydrophobic carboxy-terminal region to the isoform. The alternative splicing of Dp71 regulates its subcellular localization, with the alternatively spliced hydrophilic isoform localized to the nucleus [16]. Less is known about the localization of utrophin Up395, and its alternatively spliced isoforms Up62, Up71, and Up113. All three isoforms contain the carboxy-terminal and cysteine-rich domains, similar to their dystrophin counterparts (Figure 1) [2]. The utrophin has a ubiquitous distribution throughout the brain, and is believed to be associated with astroglial processes surrounding the microvasculature. Up113 (G-utrophin) is found in the postsynaptic density of distinct brain regions diverse from those of dystrophin. Up62 (N-utrophin) is found in glioma cells.

\section{DYSTROBREVINS}

The interaction of dystrobrevin with dystrophin occurs via one of the two helical leucine heptads of the coiledcoil region [4]. The specificity of the dystrobrevin, $\alpha$ - or $\beta$-dystrobrevin, is dependent on the isoform of dystrophin present in the tissue region. Co-immunoprecipitation studies have identified $\alpha$-dystrobrevins, in particular $\alpha$ dystrobrevin-1, as the binding partner to Dp71 in Bergmann glial cells [17]. An association with utrophin is believed to occur in perivascular astrocytes. The major $62 \mathrm{kd} \beta$ dystrobrevin isoform has been found in neurons and in the hippocampal formation, most likely associated with the fulllength dystrophin isoform [18]. Co-immunoprecipitation has revealed Dp140 as a binding partner to $\beta$-dystrobrevin, although this protein is not found in the microvasculature. An association of $\beta$-dystrobrevin with utrophin has also been demonstrated. In the cerebellum, $\beta$-dystrobrevin has been shown to be nuclear-located, probably associated with the previously undetected hydrophilic Dp71.

\section{SYNTROPHINS}

The syntrophin family of proteins are a family of closely related $59 \mathrm{kd}$ proteins containing $\mathrm{PDZ}$ domains, allowing the recruitment of signalling proteins and ion channels to the DGC. In the brain, co-precipitation of $\alpha 1$-syntrophin has been shown with neuronal dystrophin. $\beta 2$-syntrophin has a more ubiquitous expression throughout the brain, mimicking the expression of utrophin in the cerebral microvasculature [19]. Recently, two brain-specific syntrophins, $\gamma 1$ and $\gamma 2$-syntrophin, have been identified. $\gamma 1$-syntrophin has been shown to be restricted to neurons, presumably associating with dystrophin, while $\gamma 2$-syntrophin has been demonstrated to have a more ubiquitous expression in brain, and to a lesser extent in nonbrain tissue. The function of these and other syntrophins still remains unclear, although one can assume that the role is one of receptor or signalling-protein recruitment.

\section{CONCLUSIONS}

The role of dystrophin, dystrophin-like, and dystrophinassociated proteins in the molecular pathogenesis of mental 

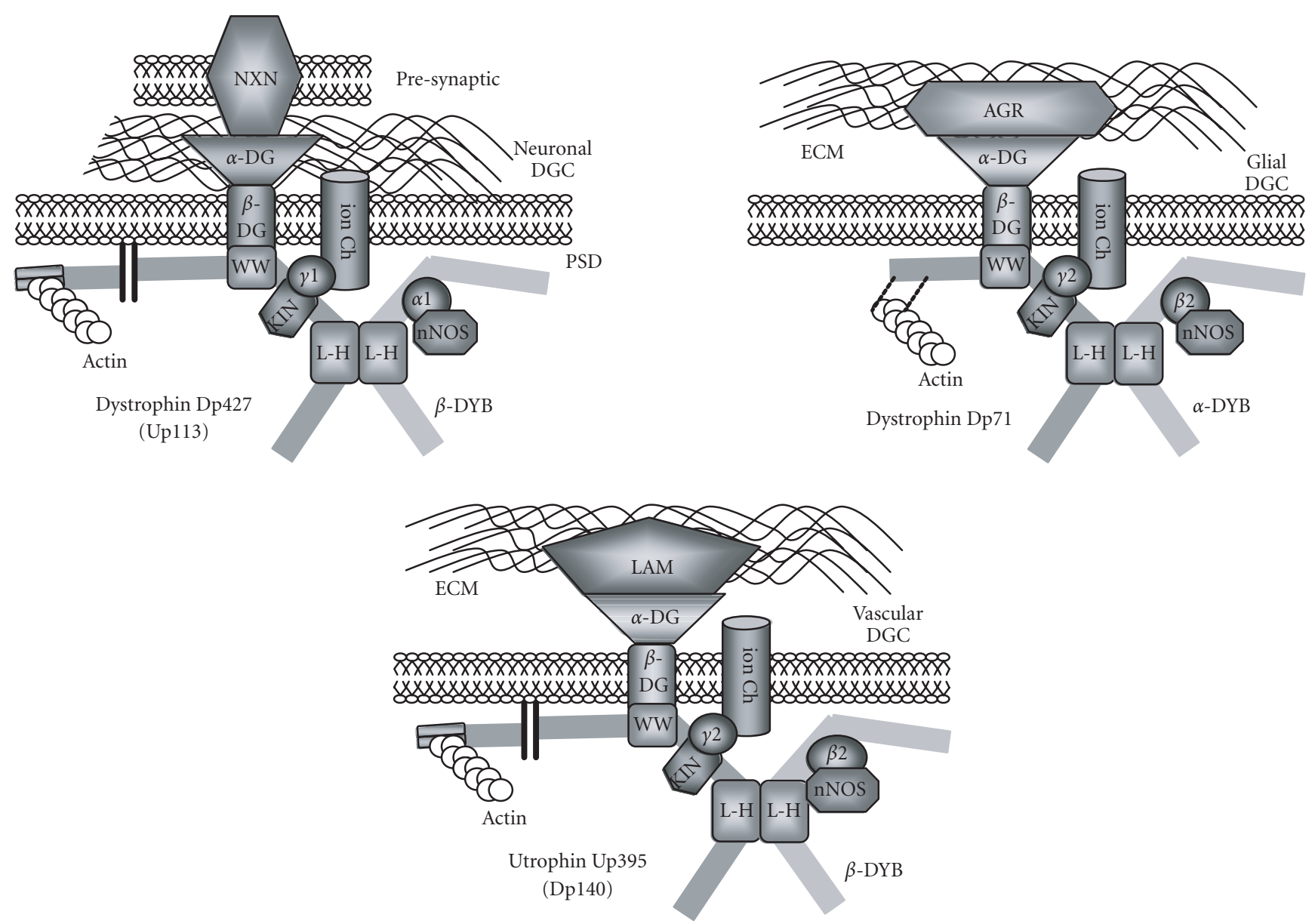

FIGURE 3. Variations in the brain dystrophin-glycoprotein complex. Schematic representation of the established members of established dystrophinglycoprotein complexes (DGC), and their associations with other peripheral proteins. Shown are a neuronal complex, a glial complex, and a general vascular complex. A dystrophin or utrophin isoform links to the $\alpha / \beta$-dystroglycan (DG) backbone and a dystrobrevin (DYB) isoform. An association with the extracellular matrix (ECM) by $\alpha$-dystroglycan is maintained through agrin (AGR) or laminin (LAM). In neurons, $\alpha$-dystroglycan is associated with neurexin (NXN), linking the presynaptic membrane (PRE) to the postsynaptic density (PSD). Other $\alpha$-dystroglycan ligands are represented by agrin (AGR) and laminin (LAM). Syntrophin isoforms $(\alpha 1, \beta 2, \gamma 1$, or $\gamma 2)$ recruit neuronal nitric oxide synthase (nNOS) to the complex, as well as other nonestablished voltage-gated ion-channels (Ion $\mathrm{Ch}$ ) and/or kinases (KIN). For abbreviations of binding domains see Figure 1.

retardation in DMD is complex, and to a large extent still elusive. Although mutations in the DMD gene are the primary defect believed to cause this phenomenon, with mutations in Dp140 [20] and Dp71 [6] implicated, it is more probable that the secondary effects of these mutations result in the specificity of the condition. The multifunctional aspect of the DGC adds to its complexity in brain. The extracellular component mediates cellular signalling through interactions of specific proteins such as laminin, merosin, perlecan, agrin, and biglycan [8]. These interactions are believed to play a role in terminal consolidation, integrity, and maintenance. Other interacting proteins, such as the newly discovered DAP neurexin [12], function to maintain cell associations, while certain viruses, such as those causing lassa fever and leprosy, utilise the complex to mediate entry to the host $[5,8]$. The cytoplasmic element of the DAPs act as adapter proteins for signalling proteins. Syntrophins and dystrobrevins recruit signalling elements such as the voltage-gated sodium channels, as well as nNOS, SAP kinase, and ErbB2 $[4,17,19]$. Syntrophins also interact with calmodulin suggesting a potential role in $\mathrm{Ca}^{2+}$-homeostasis. The cytoplasmic element of $\beta$-dystroglycan is required for the interaction of rapsyn with the nicotinic acetylcholine receptor. Recruitment of the adapter protein Grb2 also occurs [5, 8]. Evidence is mounting for the participation of the DGC in longterm memory formation. $\beta$-dystrobrevin has been shown to translocate to the nucleus, mediated by zinc finger $\mathrm{ZZ}$ domains, common amongst proteins known to migrate to the membrane from the nucleus. Recent studies have revealed a novel hydrophilic Dp71 isoform, formed through alternate splicing of the extreme carboxy-terminus [16], and may be the dystrophin isoform involved. Recruitment of signalling proteins, such as nNOS, to $\beta$-dystrobrevin has also been demonstrated. This selective recruitment to the DGC holds implications for the $\mathrm{Ca}^{2+}$-dependent cell death processes: a loss of dystrophin resulting in the cell being more susceptible to metabolic or physiological insult. This 
process would correlate with the finding that the neurons of the brains of DMD sufferers show an increase in neuronal cell death.

\section{ACKNOWLEDGMENT}

Research in the authors laboratory was supported by project grants from the Irish Health Research Board (HRB01/98) and Enterprise Ireland, Dublin (SC/2000/386).

\section{REFERENCES}

[1] Cohn RD, Campbell KP. Molecular basis of muscular dystrophies. Muscle Nerve. 2000;23:1456-1471.

[2] Culligan KG, Mackey AJ, Finn DM, Maguire PB, Ohlendieck K. Role of dystrophin isoforms and associated proteins in muscular dystrophy. Int J Mol Med. 1998;2:639-648.

[3] Blake DJ, Tinsley JM, Davies KE, Knight AE, Winder SJ, Kendrick-Jones J. Coiled-coil regions in the carboxyterminal domains of dystrophin and related proteins: potentials for protein-protein interactions. Trends Biochem Sci. 1995;20:133-135.

[4] Roberts RG. Dystrophins and dystrobrevins. Genome Biol. 2001;2(4):1-7.

[5] Winder SJ. The complexities of dystroglycan. Trends Biochem Sci. 2001;26:118-124.

[6] Mehler MF. Brain dystrophin, neurogenetics, and mental retardation. Brain Res Brain Res Rev. 2000;32:277307.

[7] Bresolin N, Castelli E, Comi GP, et al. Cognitive impairment in Duchenne muscular dystrophy. Neuromuscul Disord. 1994;4:359-369.

[8] Henry MD, Campbell KP. Dystroglycan inside and out. Curr Opin Cell Biol. 1999;11:602-607.

[9] Ramjeesingh M, Huan LJ, Garami E, Bear CE. Novel method for evaluation of the oligomeric structure of membrane proteins. Biochem J. 1999;342:119-123.

[10] Culligan K, Glover L, Dowling P, Ohlendieck K. Brain dystrophin-glycoprotein complex: persistent expression of $\beta$-dystroglycan, impaired oligomerization of Dp71 and up-regulation of utrophins in animal models of muscular dystrophy. BMC Cell Biol. 2001;2(1):2.

[11] McDearmon EL, Combs AC, Ervasti JM. Differential vicia villosa agglutinin reactivity identifies three distinct dystroglycan complexes in skeletal muscle. J Biol Chem. 2001;276:35078-35086.

[12] Sugita S, Saito F, Tang J, Satz J, Campbell KP, Sudhof TC. A stoichiometric complex of neurexins and dystroglycan in brain. J Cell Biol. 2001;154:435-445.

[13] Blake DJ, Hawkes R, Benson MA, Beesley PW. Different dystrophin-like complexes are expressed in neurons and glia. J Cell Biol. 1999;147:645-658.

[14] Chelly J, Hamard G, Koulakoff A, Kaplan JC, Kahn A, Berwald-Netter Y. Dystrophin gene transcribed from different promoters in neuronal and glial cells. Nature. 1990;344:64-65.
[15] Austin RC, Morris GE, Howard PL, Klamut HJ, Ray PN. Expression and synthesis of alternatively spliced variants of Dp71 in adult human brain. Neuromuscul Disord. 2000;10:187-193.

[16] Gonzalez E, Montanez C, Ray PN, et al. Alternative splicing regulates the nuclear or cytoplasmic localization of dystrophin Dp71. FEBS Lett. 2000;482:209-214.

[17] Ueda H, Baba T, Terada N, et al. Immunolocalization of dystrobrevin in the astrocytic endfeet and endothelial cells in the rat cerebellum. Neurosci Lett. 2000;283:121124.

[18] Peters MF, O’Brien KF, Sadoulet-Puccio HM, Kunkel LM, Adams ME, Froehner SC. Beta-dystrobrevin, a new member of the dystrophin family. Identification, cloning, and protein associations. J Biol Chem. 1997;272:31561-31569.

[19] Gorecki DC, Abdulrazzak H, Lukasiuk K, Barnard EA. Differential expression of syntrophins and analysis of alternatively spliced dystrophin transcripts in the mouse brain. Eur J Neurosci. 1997;9:965-976.

[20] Bardoni A, Felisari G, Sironi M, et al. Loss of Dp140 regulatory sequences is associated with cognitive impairment in dystrophinopathies. Neuromuscul Disord. 2000;10:194-199.

\footnotetext{
* Corresponding author.

E-mail: kay.Ohlendieck@ucd.ie

Fax: +353 1 2692749; Tel: +35317161503
} 

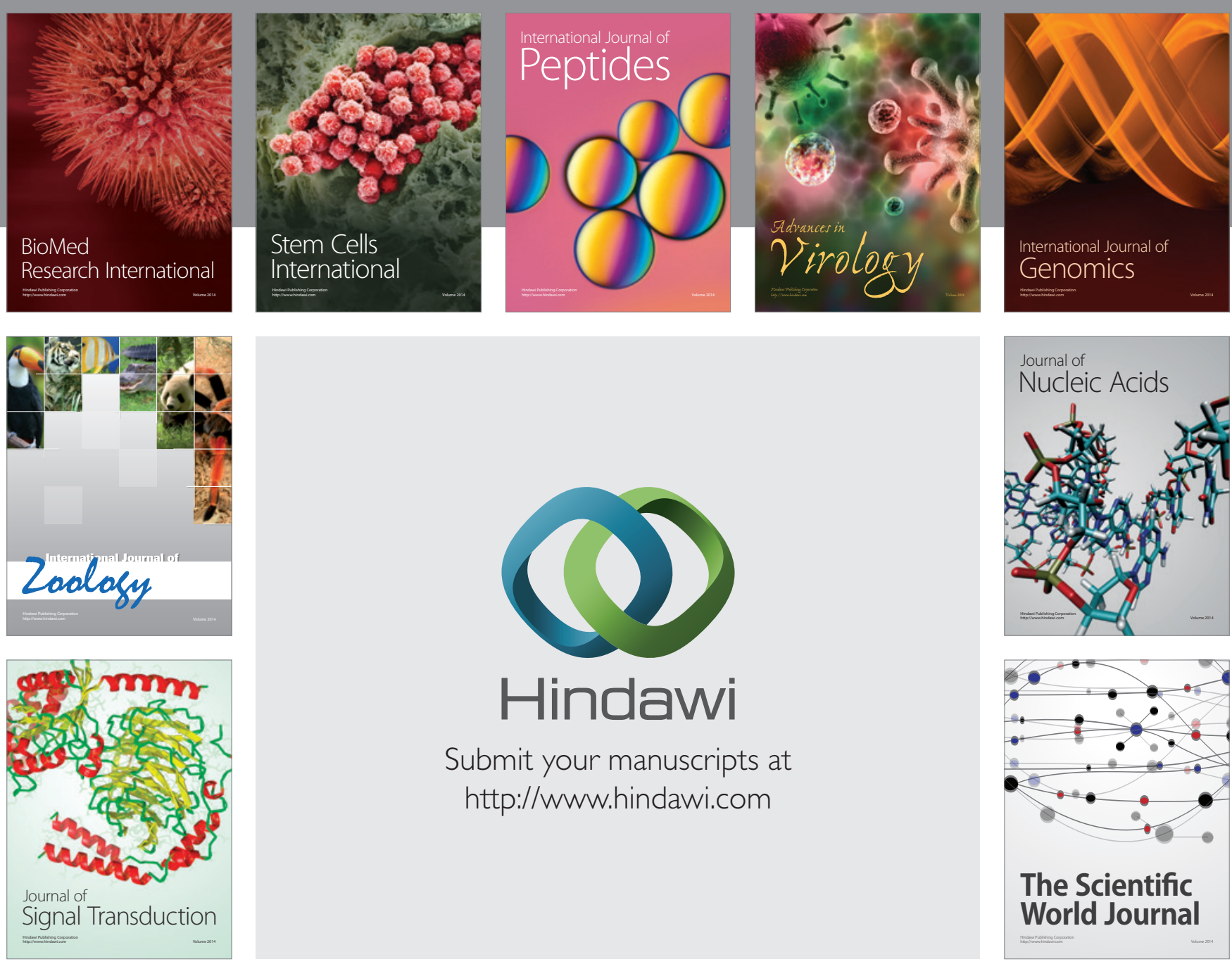

Submit your manuscripts at

http://www.hindawi.com
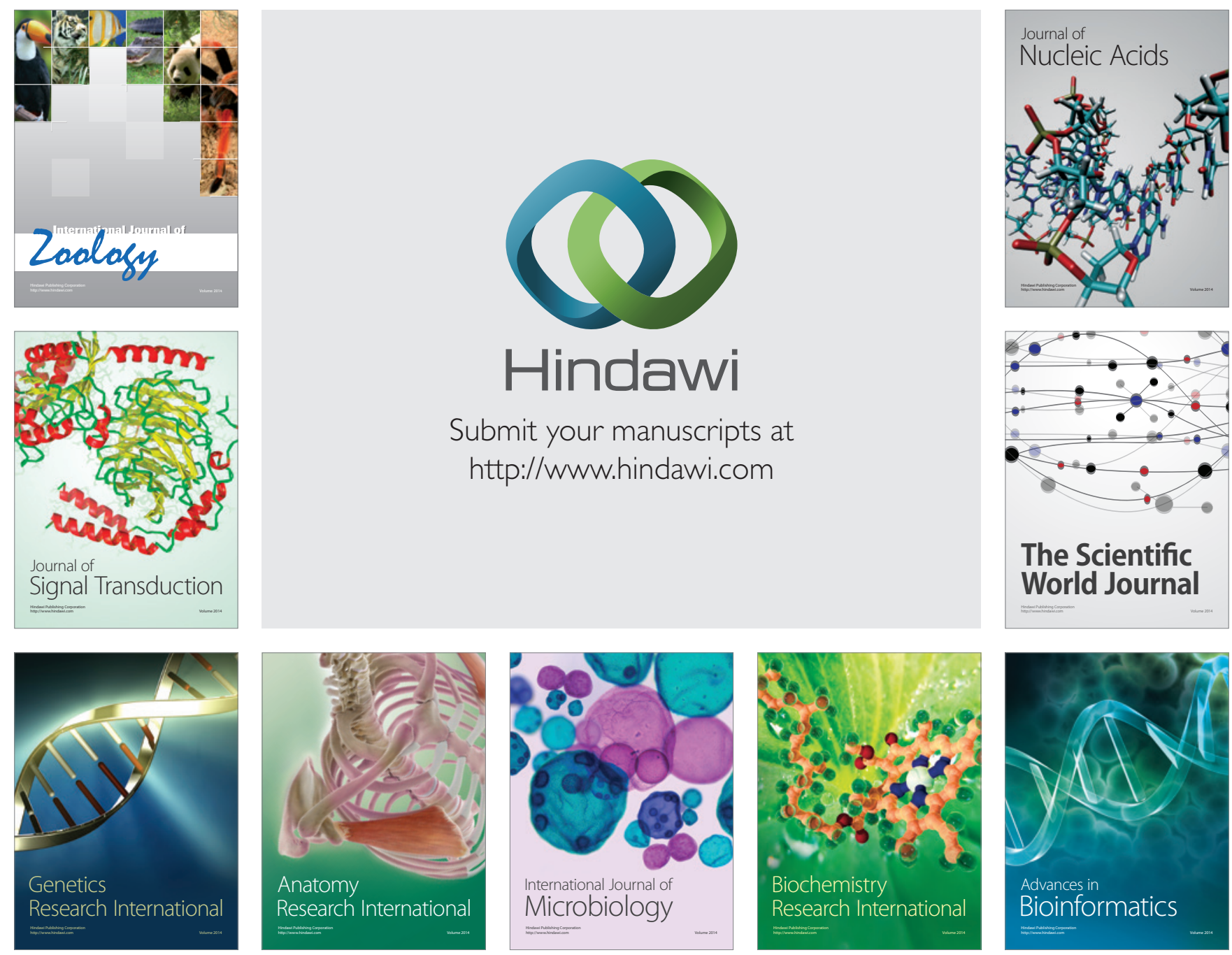

The Scientific World Journal
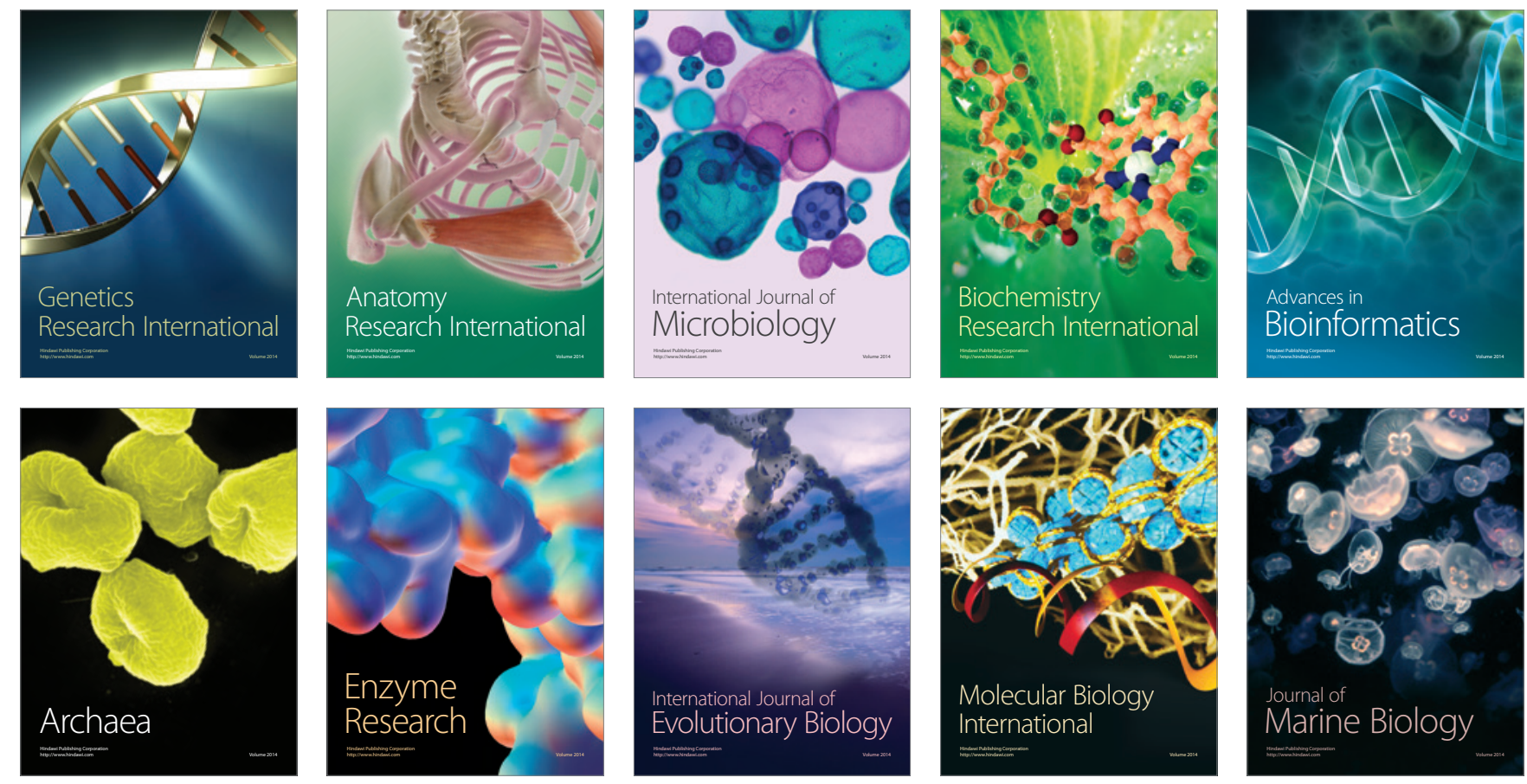